\title{
Review of: "Serine 1283 in extracellular matrix glycoprotein Reelin is crucial for Reelin's function in brain development"
}

shengzhou wu

Potential competing interests: The author(s) declared that no potential competing interests exist.

Reelin,acting upon Reelin receptors including ApoER2 and VLDLR, plays role in neuronal migration,synapse function, and lamination of the cerebral and cerebellar cortex. Reelin contained 8 reelin repeats and cleavage at its N-terminal virtually abolishes Dab1 phosphorylation. In addition, Reelin is a serine protease of extracellular matrix such as targeting fibronectin and cleave adhesion molecues. Casein kinase2(CK2), expressed in the brain, mediates L1 adhesion molecules, promote neurite development and neuronal survival by decreased the levels of p53 and PTEN. CK2 has been demonstrated to phosphorylate Reelin.

In this manuscript, the authors found:1) Mutation of serine at 1283 in Reelin entails a reeler-like phenotype in mice including displaced cytoarchitecture of the cerebral and cerebellar cortex and the hippocampus.

The mice also indicated motor deficit. 2)Cajal-Retzius cells,localizing in the outmost layer of the cortex and synthesizing Reelin protein, were misplaced due to this mutation. 3) In rln ${ }^{\mathrm{A} / \mathrm{A}}$ mutant, full-length Reelin protein was reduced in postnatal brain homogenates. 4)By in utero electroporation to deliver N-R6 partially restore the neuronal migration in $\mathrm{r} / \mathrm{n}^{\mathrm{A} / \mathrm{A}}$. 5)Further, they indicated that inhibition of CK2 activity decreased Reelin stability. 6)Reelin did not cleave L1 and the co-purified MBP did.

Overall,the study is delicately designed.

Questions:

1,in Fig.7b,IP with Reelin antibody G10 was able to capture N-R2 with phosphor-serine antibody,indicating that phosphorylation also occurred at N-terminal of Reelin in addition to the phosphorylation at serine1283 on R3. The possible additional data should take advantage of cerebellar culture from $\mathrm{rln} \mathrm{n}^{\mathrm{A} / \mathrm{A}}$ mice. With this culture, it is interesting to find out whether addition of TBB increased the stability of Reelin.

2 , It is interesting to directly demonstrate whether degradation of S/A mutated Reelin was increased by ADAMTS-4.

3,In this study, Reelin was shown not to degrade its binding partner,L1. Reelin is shown to interact with serine protease MBP-1 which is expressed at the end of neuronal migration. Therefor, how mutated Reelin affect neuronal migration is yet unclear. 
